\title{
Low Frequency Core Losses Components of FeNiMo Powder Compacted Materials
}

\author{
D. OleKŠÁKOVÁ ${ }^{a, *}$, P. KOLlÁR ${ }^{b}$ AND J. FÜZER ${ }^{b}$ \\ ${ }^{a}$ Institute of Manufacturing Management, Faculty of Manufacturing Technologies, Technical University of Košice, \\ Bayerova 1, 08001 Prešov, Slovakia \\ ${ }^{b}$ Institute of Physics, Faculty of Science, P. J. Šafárik University, Park Angelinum 9, 04154 Košice, Slovakia
}

\begin{abstract}
The relations of core losses with the frequency of FeNiMo alloys were investigated. The core energy losses were determined by the measurements of $\mathrm{dc}$ and ac hysteresis loops as functions of frequency $(1 \mathrm{~Hz}-50 \mathrm{~Hz})$. The usual three-component concept of separation of core losses consisting of hysteresis, eddy current and anomalous losses was used to explain the influence of the powder particle size on core loss frequency dependences.
\end{abstract}

DOI: 10.12693/APhysPolA.133.639

PACS/topics: $75.50 . \mathrm{Vv}, 85.70 .-\mathrm{w}, 75.50 . \mathrm{Bb}$

\section{Introduction}

Most remarkable permalloys are the alloys with composition close to $\mathrm{Fe}_{20} \mathrm{Ni}_{80}$, where both anisotropy and magnetostriction fall to zero at almost the same composition. A tendency towards unfavourable $\mathrm{Ni}_{3} \mathrm{Fe}$-type $\mathrm{L}_{2}$ atomic ordering can be suppressed by Mo addition, i.e., in supermalloy FeNiMo [1].

The appropriate structure of the supermalloys (produced usually in the form of thin sheet) having initial permeability much larger than that of pure iron arises after proper heat treatment. The form of a sheet is not suitable for some applications and therefore it is logical to attempt to prepare such material in another form, for example as a cylinder or a ring, which would be more convenient for construction of some type components for electronic devices. One of the suitable methods for preparation of 3 -D samples is compaction of the powder prepared by mechanical milling or mechanical alloying [2]. Mechanical milling is a useful powder processing technique that can produce a variety of equilibrium and non-equilibrium alloy phases $[2-5]$. Some researchers reported results concerning FeNiMo powders system produced by mechanical milling in $[6,7]$.

In addition to our previous study on $\mathrm{Fe}-\mathrm{Ni}[8]$ and on $\mathrm{Fe}-\mathrm{Ni}-\mathrm{Mo}$ [3], now we focused the interest on low frequency losses components of FeNiMo powder compacts.

\section{Experimental}

The $\mathrm{Fe}_{16} \mathrm{Ni}_{79} \mathrm{Mo}_{5}$ (wt.\%) swarfs were prepared from the ingot of the same chemical composition using a lathe (while it was not possible to prepare the thin ribbon by melt spinning in contrast with binary $\mathrm{Fe}_{19} \mathrm{Ni}_{81}$ (wt. \%) alloy, which was successfully prepared by melt spinning

*corresponding author; e-mail: denisa.oleksakova@tuke.sk in the form of thin ribbon as described in $[7,9])$. The swarfs were milled using a RETSCH PM4000 planetary ball mill with hardened steel vials for 1 and 100 hours. To make the alloy more brittle (and thus more suitable for milling), the bowl together with milled material was cooled before grinding by diving in liquid nitrogen. The rotational speed of main mill's disk was $180 \mathrm{rpm}$ and ball to powder ratio was of $6: 1$.

The powder samples were compacted at a pressure of $800 \mathrm{MPa}$, the temperature of $600^{\circ} \mathrm{C}$ (causing the best magnetic properties when annealed in the range from $400^{\circ} \mathrm{C}$ to $600{ }^{\circ} \mathrm{C}$ ) was stabilized for $5 \mathrm{~min}$. The resulting cylinders have following parameters: diameter of $10 \mathrm{~mm}$, height of approx. $2.5 \mathrm{~mm}$. To prepare the ring samples we drilled an axial hole with a diameter of $5 \mathrm{~mm}$ into the centre the cylinders.

- FeNiMo 1 - a bulk sample prepared by the warm consolidation of the swarfs milled for $1 \mathrm{~h}$.

- FeNiMo 100 - a bulk sample prepared by the warm consolidation of the swarfs milled for $100 \mathrm{~h}$.

More details concerning the milling and compaction process of FeNiMo alloy and its magnetic characteristics have been already reported in our previous articles $[9,10]$.

The dc hysteresis loops were obtained by a hysteresisgraph based on the fluxmeter at maximum induction of $0.2 \mathrm{~T}$, by the point-by-point (ballistic) method, with $4 \mathrm{~s}$ delays between two measured points. The ac hysteresis loops were measured in the frequency range from 1 $\mathrm{Hz}$ to $50 \mathrm{~Hz}$. We have determined the hysteresis losses $W_{d c}$, core losses $W_{t}$ and coercive field directly from the measured hysteresis loops.

\section{Coercivity}

As proved earlier, the coercivity $H_{c}$ decreases with increasing annealing temperature (from $800^{\circ} \mathrm{C}$ to $1100^{\circ} \mathrm{C}$ ), reaching a minimum value of $11.2 \mathrm{~A} / \mathrm{m}$ for FeNiMo bulk sample annealed at $1100{ }^{\circ} \mathrm{C}[7,9]$ and it is comparable to 
those for the material of the same composition produced by casting in the form of a thin sheet [11].

The coercive field (determined from hysteresis loops with $B_{m}=0.2 \mathrm{~T}$ ) as a function of the frequency for bulk samples prepared by the compaction the powder milled for 1 and 100 hours is shown the Fig. 1 a). We can see that the bulk sample prepared by the powder milled longer time reaches higher values of the coercive field in whole measured frequency range. In first part of the frequency range approx. from $1 \mathrm{~Hz}$ to $5 \mathrm{~Hz}$ the most significant effect for higher coercive field of sample FeNiMo 100 is the influence of more defects introduced to the powder sample during mechanical milling acting as pinning centres for domain wall displacement. Morever, the influence of particle size and shape may be a reason of the shape of dc hysteresis loops (measured at $B_{m}=$ $0.2 \mathrm{~T}$ ) in Fig. 2a) and b). The tendency to saturate the dc hysteresis loop (measured at maximum magnetic field of $4800 \mathrm{~A} / \mathrm{m}$ ) for the sample FeNiMo 1 is significantly stronger (Fig. 2 c)) than that for the sample FeNiMo 100 (Fig. 2 d)) what confirms the assumption of the influence of the defects introduced during the milling process.
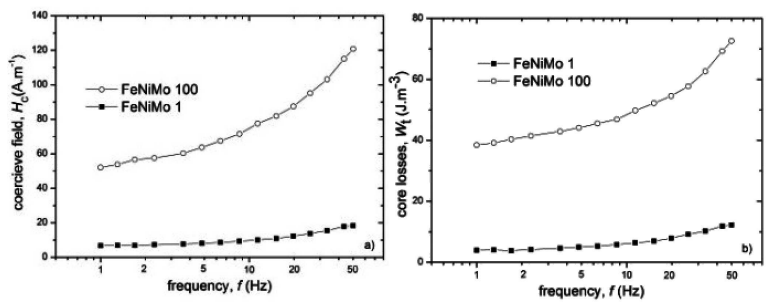

Fig. 1. Coercive field a) and core losses b) as a function of frequency measured for bulk FeNiMo prepared by the compaction of the powder milled for $1 \mathrm{~h}$ and $100 \mathrm{~h}$.
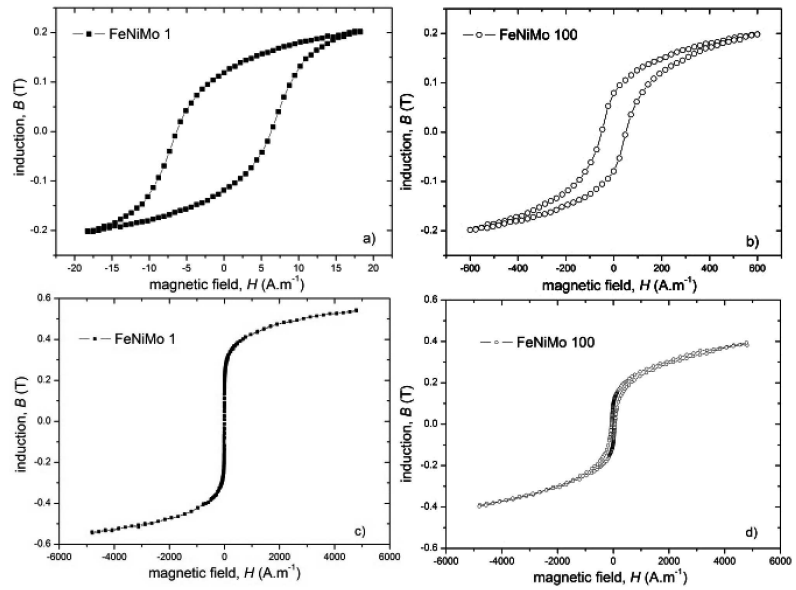

Fig. 2. The dc hysteresis loops of samples a) FeNiMo 1, b) FeNiMo 100 measured at $B_{m}=0.2 \mathrm{~T}$ and dc hysteresis loops of samples c) FeNiMo 1, d) FeNiMo 100 measured at $H_{c}=4800 \mathrm{~A} / \mathrm{m}$.

In the second part of the frequency range $5-50 \mathrm{~Hz}$, the function of the coercive field versus frequency (Fig. 1a)) has the slope of coercivity for the FeNiMo 100 sample higher value than that for FeNiMo 1 sample. It can be explained by the prevalence of magnetization vector rotation as a dominant magnetization process in smaller powder elements (sample FeNiMo 100) leading to the larger increase of the hysteresis loop area (core losses).

\section{Core losses}

The tendency for the frequency dependence of the total losses qualitatively follows the coercive field function for both samples.

The specific energy core losses $W_{t}\left[\mathrm{~J} / \mathrm{m}^{3}\right]$ can be expressed as

$$
W_{t}=W_{d c}+W_{c l}+W_{a},
$$

where $W_{d c}$ - hysteresis losses, $W_{c l}$ - classical eddy current losses and $W_{a}$ - anomalous losses $[11,12]$. Hysteresis losses $W_{d c}$ can be calculated as an area of dc hysteresis loops and is described as coefficient $C_{d c}, W_{d c}=C_{d c}$.

For eddy current losses $W_{c l}$ is usually taken into account is the expression [12]

$$
W_{c l}=\frac{\pi^{2} B_{\max }^{2} d^{2}}{\beta} \sigma f=C_{c l} f,
$$

where $C_{c l}=\frac{\pi^{2} B_{\max }^{2} d^{2}}{\beta} \sigma$ is frequency proportionality coefficient, which include material parameters $(d$ - thickness of the sample and $\sigma$-conductivity of the ferromagnetic material) and measurements conditions $\left(B_{m}\right.$ - maximum induction and $\beta$-geometrical coefficient of the cross section sample perpendicular to the magnetic induction). For the geometrical coefficient $\beta$ of the sample with the width $w$ and the height $h$ of the rectangle is valid [12]:

$$
\beta=\frac{6}{1-0.633\left(\frac{w}{h}\right) \tanh \left(1.58 \frac{h}{w}\right)} .
$$

For the anomalous losses coming from damping of domain walls displacement of local eddy we can write the expression [12]

$$
W_{a}=\frac{8.8 \sqrt{S B_{\max }^{3} G \sigma V_{0}}}{\rho}=C_{a} \sqrt{f},
$$

where $C_{a}=\frac{8.8 \sqrt{S B_{\max }^{3} G \sigma V_{0}}}{\rho}$ is frequency proportionality coefficient, which includes parameters of the material and measurements conditions ( $S$ - the cross section of the material perpendicular to the magnetic induction, $G$ - parameter depending on the material, $V_{0}$ - parameter that depend on the magnetisation and the material).

According to the formulas (1), (2) and (3) we can suppose that the frequency dependences of the core losses for all samples is

$$
W_{t}=W_{d c}+W_{c l}+W_{a}=C_{d c}+C_{c l} f+C_{a} f^{\frac{1}{2}} .
$$

Fig. $1 \mathrm{~b}$ ) shows the core losses as a function of frequency of both samples in the frequency range from $1 \mathrm{~Hz}$ to 50 $\mathrm{Hz}$ measured at $B_{m}=0.2 \mathrm{~T}$.

We suppose the presence of all three contributions to the total losses: hysteresis, eddy current and anomalous. The size of the contributors to the total loses was performed by the fitting the experimental results according to the Eq. 5, which allowed to calculate the coefficients 
$C_{d c}, C_{c l}$ and $C_{a}$, and specific conductivity $\sigma$ according to Eq. 2. and Table I.

TABLE I

Measured and calculated parameters of FeNiMo samples.

\begin{tabular}{c|c|c}
\hline \hline Sample & FeNiMo 1 & FeNiMo 100 \\
\hline powder milling time $[\mathrm{h}]$ & 1 & 100 \\
exp. values of $W_{d c}\left[\mathrm{~J} / \mathrm{m}^{3}\right]$ & 3.5 & 38.6 \\
$C_{d c}\left[\mathrm{~J} / \mathrm{m}^{3}\right]$ & 3.4 & 38.4 \\
$C_{c l}\left[\mathrm{~J} /\left(\mathrm{m}^{3} \mathrm{~s}\right)\right]$ & 0.12 & 0.46 \\
$C_{a}\left[\mathrm{~J} / \mathrm{m}^{3} \mathrm{~s}^{1 / 2}\right]$ & 0.49 & 1.57 \\
$\sigma[\mathrm{S} / \mathrm{m}]$ & $4.55 \times 10^{5}$ & $1.07 \times 10^{6}$
\end{tabular}

The values of hysteresis losses $W_{d c}$ calculated from frequency dependences of core energy losses $W_{t}$ are close to the values $W_{d c}$ obtained by measuring of dc hysteresis loops.
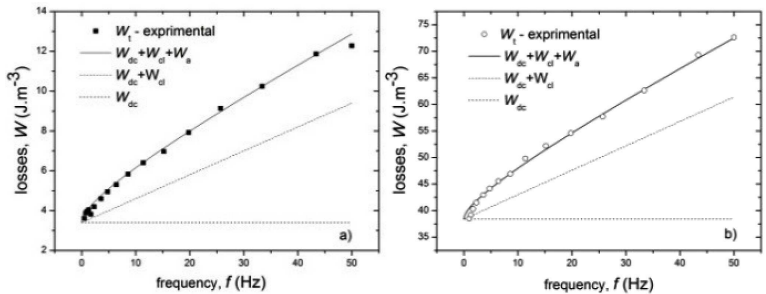

Fig. 3. The frequency dependence of the contributions to the core losses from dc to $50 \mathrm{~Hz}$ at $B_{m}$ at $0.2 \mathrm{~T}$ calculated by fitting according to Eq. (4), compared with experimental results a) for sample FeNiMo 1 and b) for sample FeNiMo 100 .

All components to the total losses are higher for the sample FeNiMo 100 prepared by compaction of powder milled for a significantly longer time than that for the sample FeNiMo 1, Fig. 3. The larger value of hysteresis loss is caused by introduction of defects at milling (similar as for coercivity and coercive field). The sample FeNiMo 100 exhibits larger value of specific conductivity (smaller particles fit very well to each other after compaction and are responsible for better electric contact) leading to the steeper frequency dependence of eddy current losses. The restoring the magnetic "contact" between two particles (confirmed by the increase of specific conductivity) causes the increase of the anomalous component of losses in FeNiMo 100 more significant. The higher influence of anomalous losses in FeNiMo 100 is probably caused by lower number of movable domains, performing Barkhausen jumps on longer distances overtaking attractive forces of pinning centres created as a consequence of longer milling process walls in comparison with FeNiMo 1.

\section{Conclusions}

The bulk sample FeNiMo1 prepared by the compaction of powder milled for 1 hour exhibits lower values of core losses and all their contributions (hysteresis losses, eddy current losses, anomalous losses) in comparison with the sample prepared by compaction of FeNiMo 100 hours milled powder. For lower value of hysteresis loss is responsible easier movement of domain walls in ferromagnet with lower concentration of defects. Lower specific conductivity of bulk material consisting of larger better insulated particles (FeNiMo 1) leads to the lower values of eddy current and anomalous losses than in sample consisting of compaction longer milled powder (FeNiMo 100).

\section{Acknowledgments}

This work was supported by the Slovak Research and Development Agency under contract No. APVV-15-0115 and by the Scientific Grant Agency of the Ministry of Education of the Slovak Republic and the Slovak Academy of Sciences, VEGA 1/0330/15 and VEGA 1/0377/16.

\section{References}

[1] J.M.D. Coey, Magnetism and Magnetic Materials, Cambridge University Press, 2010, pp. 449-540.

[2] C. Suryanarayana, Progress in Mater. Sci. 46, 1 (2001).

[3] J. Füzer P. Kollár, D. Olekšáková, S. Roth, J. Alloys Compd. 483, 557 (2007).

[4] L. Karimi et al., J. Alloys Compd. 509, 6571 (2011).

[5] T.F. Marinca, B.V. Neamţu, F. Popa, V.F Tarţa, P. Pascuta, A.F. Takacs, I. Chicinas, Appl. Surf. Science 285, 2 (2013).

[6] M. Karolus, E. Jartych, D. Oleszak, Acta Phys. Pol. A 102, 253 (2002).

[7] D. Olekšáková, P. Kollár, J. Füzer, M. Kusý, Acta Phys. Pol. A 113, 75 (2008).

[8] D. Olekšáková, J. Füzer, P. Kollár, S. Roth, J. Magn. Magn. Mater. 333, 18 (2013).

[9] D. Olekšáková, P. Kollár, J. Füzer, M. Kusý, S. Roth, K. Polanski, J. Magn. Magn. Mater. 316, e 838 (2007).

[10] J. Füzer P. Kollár, D. Olekšáková, S. Roth, Acta Phys. Pol. A 113, 59 (2008).

[11] B.D. Cullity, C.D. Graham, Introduction to Magnetic Materials,Wiley, IEEE Press, Piscataway, NJ 08854, 2008.

[12] H. Kronmüller, S. Parkin, Handbook of Magnetism and Advanced Magnetic Materials, Micromagnetism 2, Wiley, New York 2007. 